\title{
A New Method of Determining the Characteristics of Evolved Binary Systems Revealed in the Observed Circumstellar Patterns: Application to AFGL 3068
}

\author{
Hyosun $\mathrm{Kim}^{1}$ and Ronald E. Taam ${ }^{1,2}$
}

\begin{abstract}
The binary characteristics of asymptotic giant branch (AGB) stars are imprinted in the asymmetric patterns of their circumstellar envelopes (CSEs). We develop a simple method for constraining the orbital parameters of such binary stars from the characteristics of a spiral-like pattern observed at large distances from the central stars. We place constraints on the properties of AFGL 3068 (i.e., the masses of binary components, the viewing inclination of the orbital plane, as well as the orbital period, velocity, and separation). In particular, the mass of the companion star of AFGL 3068 is estimated to be greater than $2.6 M_{\odot}$. This method is applicable to other AGB stars, providing a step toward understanding the role binary stars can play in explaining the diverse patterns in observed CSEs.
\end{abstract}

Subject headings: binaries: general — circumstellar matter — stars: AGB and post-AGB — stars: carbon — stars: late-type — stars: winds, outflows

\section{INTRODUCTION}

At the end of a star's life, low- to intermediate-mass stars return their mass to interstellar space by means of slow, dense winds (Habing 1996) during the AGB phase. The material expelled from these evolved stars can take the form of irregular/regular patterns and shapes prior to mixing with the interstellar medium. The dense CSEs often show strong asymmetries in the late stellar evolutionary stages, whose origin probably lies in their progenitor systems. Recent studies reveal that a binary system may play a crucial role in forming the various

\footnotetext{
${ }^{1}$ Academia Sinica Institute of Astronomy and Astrophysics, P.O. Box 23-141, Taipei 10617, Taiwan; hkim@asiaa.sinica.edu.tw

${ }^{2}$ Department of Physics and Astronomy, Northwestern University, 2131 Tech Drive, Evanston, IL 60208; r-taam@northwestern.edu
} 
shapes of CSEs (Huggins 2007). However, the determination of the system properties of a wide binary is challenging.

AFGL 3068 is the first AGB binary system that clearly shows a wide spiral pattern. Two point-like sources in near-infrared images have been revealed using the Keck adaptive optics (Morris et al. 2006), making AFGL 3068 an evolved stellar system in which the binary components are indeed detected. Based on a distance of $1 \mathrm{kpc}$, the projected separation is $109 \mathrm{AU}$, which is relatively large to infer a direct binary interaction. Nevertheless, AFGL 3068 exhibits evidence of a binary interaction in view of the large scale spiral pattern within its CSE extending over 10,000 AU in dust-scattered Galactic light (Mauron \& Huggins 2006). This spiral pattern provides support for the role of a companion star in shaping the CSE (Soker 1994; Mastrodemos \& Morris 1999; He 2007; Edgar et al. 2008), necessitating an attempt to constrain binary properties from the large scale density pattern (Kim 2011; Kim \& Taam 2012a, b).

In a parameter study of hydrodynamical simulations on the asymmetric circumstellar pattern due to the binary motions (Kim \& Taam 2012b, KT hereafter), we have shown the directional dependence of the wind flow blown out from the AGB star. It reflects the difference between the line-of-sight velocity of the flow and the propagation speed of the pattern in the plane of the sky. The pattern propagation speed is $\left\langle V_{w}\right\rangle+2 V_{p} / 3$ throughout the orbital plane, but equals to $\left\langle V_{w}\right\rangle$ in the vertical direction, resulting in an overall oblate shape. This shape is characterized by the aspect ratio $a / b$ corresponding to $\left(<V_{w}>\right.$ $\left.+2 V_{p} / 3\right) /<V_{w}>$, which appears as an ellipse with inclination. In addition, the maximum fluctuation of the gas velocity relative to the average wind velocity value $\left\langle V_{w}\right\rangle$ is less than the orbital velocity $V_{p}$ of the mass-losing star.

Based on these findings, we propose a new method to constrain the binary properties from four observed quantities corresponding to the projected axial ratio $(a / b)_{\text {proj }}$, the projected binary separation $\left(r_{p}+r_{\text {comp }}\right)_{\text {proj }}$, the position angle of the star from the major axis of the elliptical shape (PA), and the arm spacing $\Delta r_{\text {arm }}$. Here, $r_{p}$ and $r_{\text {comp }}$ correspond to the separation of the AGB star and its companion from the center of mass of the system. For the first application of this method, we place constraints on AFGL 3068, as an extreme carbon star (Lebofsky \& Rieke 1977; Jones et al. 1978; Silverglate et al. 1979; Jewell \& Snyder 1982; Volk et al. 1992; Zhang et al. 2009), at the tip of the AGB. Figure1 shows the HST image of AFGL 3068, overlaid with our binary model with the mass ratio of 1.13. 

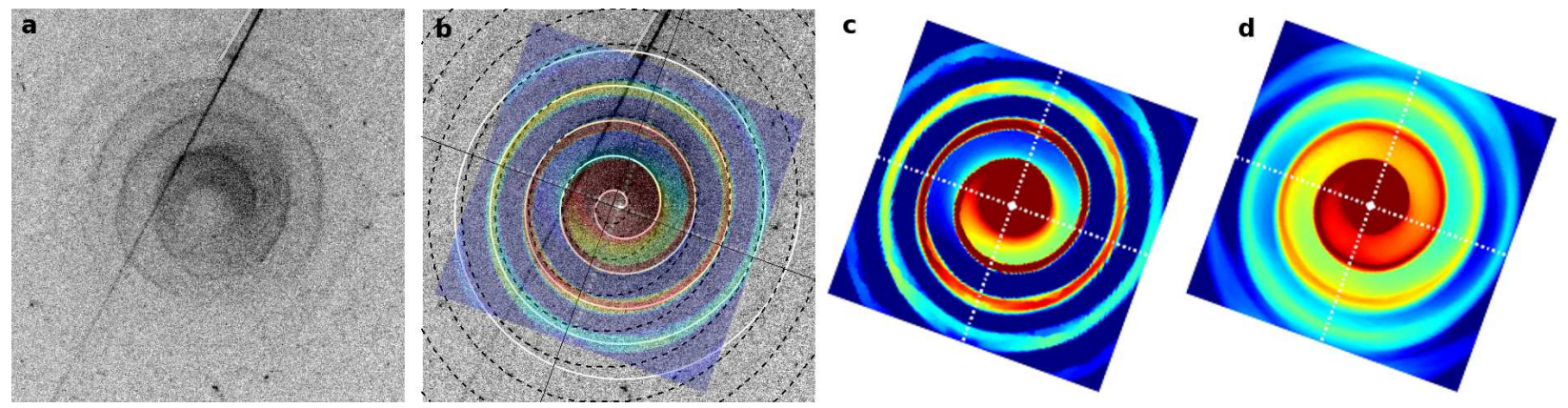

Fig. 1.- (a) Image of AFGL 3068 in the wide-V F606W filter of the Hubble Space TelescopeACS camera with the field size of 25".6 × 25".6 (Credit: Mauron \& Huggins, A\&A, 452, 257, 2006, reproduced with permission (C) ESO.). North is up, and east is left. The bright straight line refers to a diffraction spike of a star in the field of view of the original image. (b) Same image with an Archimedes spiral fit (Mauron \& Huggins 2006), overlaid with our three-dimensional hydrodynamic model. The observed pattern is more elongated than an Archimedes spiral having a constant spacing, particularly in the northwest direction. The elongated shape of the spiral pattern is described by the ellipses with the axial ratio of 1.1 corresponding to the eccentricity of 0.417 (dashed lines). The ellipses have the major axis (the intersection of the plane of the sky; line of nodes) at $70^{\circ}$ counterclockwise from eastwest, where the binary stars are aligned at the center (Morris et al. 2006), and the minor axis is perpendicular to the major axis (black thin lines). The difference in the semi-major radius between the dashed lines is $2350 \mathrm{AU}$ at a distance of $1 \mathrm{kpc}$. (c) Density distribution at the mid-plane in the line of sight of a hydrodynamic model for binary stars composed of a mass-losing star $\left(3.5 M_{\odot}\right)$ and a main-sequence star $\left(3.1 M_{\odot}\right)$ with the orbital separation of $166 \mathrm{AU}$. The corresponding orbital velocities are $2.8 \mathrm{~km} \mathrm{~s}^{-1}$ and $3.2 \mathrm{~km} \mathrm{~s}^{-1}$, respectively. The wind velocity varies about a mean value of $11.5 \mathrm{~km} \mathrm{~s}^{-1}$. An adiabatic index $\gamma$ of 1.4 is employed to produce a temperature profile of the form $r^{-0.83}$ as obtained from the CO molecule excitation analysis (Woods et al. 2003). The orbital plane is inclined by $50^{\circ}$ from the plane of the sky. (d) Column density distribution. 


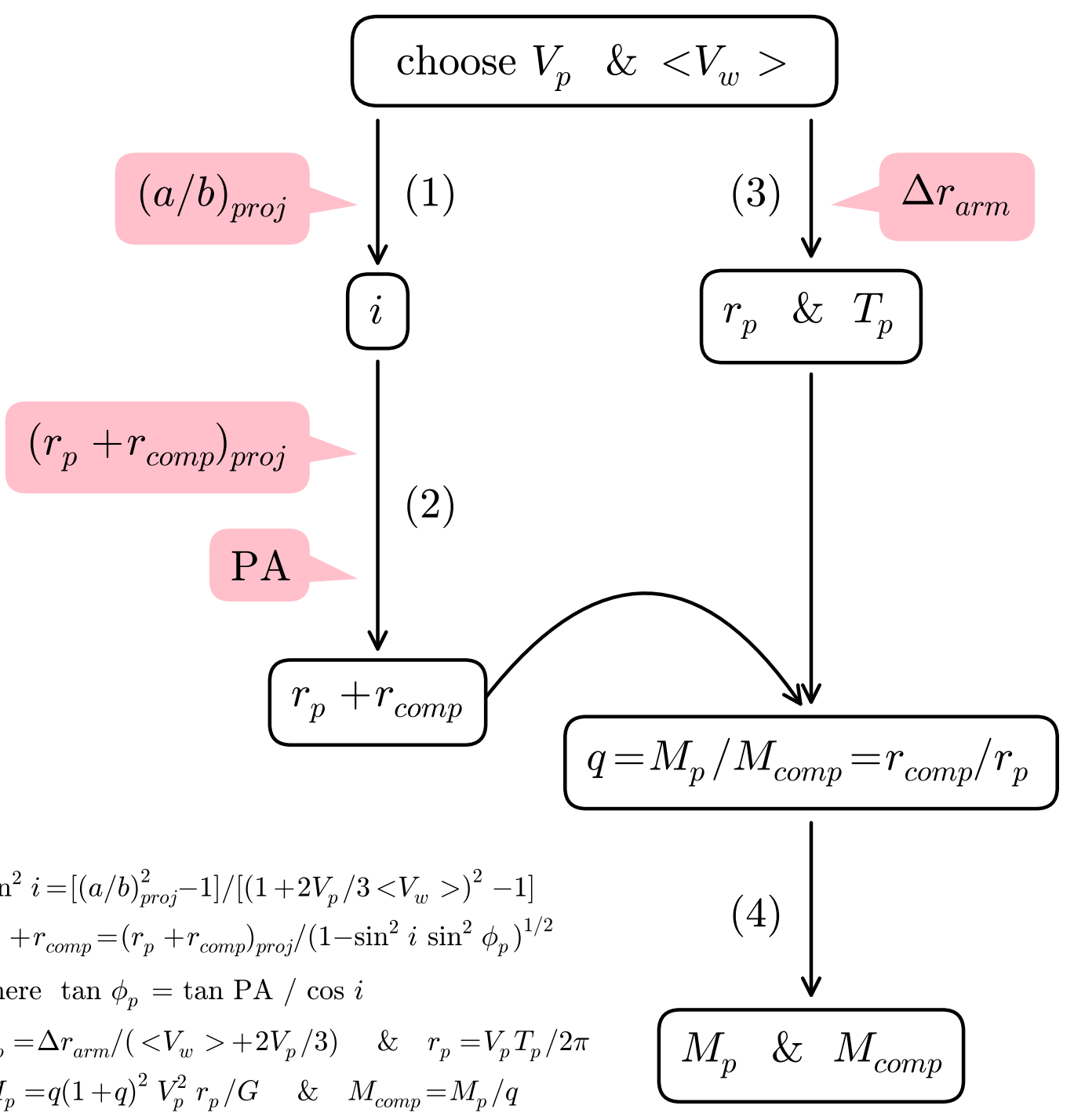

Fig. 2.- Schematic diagram for the process of deriving binary properties (open boxes) from four observables (pink boxes), based on a parameter study using hydrodynamic simulations (KT). The speeds of orbital motion $V_{p}$ and wind motion $\left\langle V_{w}\right\rangle$ of the mass-losing star are considered as the model parameters. The four required observables correspond to the projected major-minor axial ratio of the ellipse characterizing the overall shape of the pattern $(a / b)_{\text {proj }}$, the projected orbital separation $\left(r_{p}+r_{\text {comp }}\right)_{\text {proj }}$, the position angle of the stars with respect to the major axis (line of nodes), and the spiral arm spacing $\Delta r_{\text {arm }}$. The result of the analysis leads to the masses $M_{p}$ and $M_{\text {comp }}$, orbital radii $r_{p}$ and $r_{\text {comp }}$ for the mass-losing star and the companion, respectively, the orbital period $T_{p}$, and the inclination angle of the orbital plane $i$. 


\section{NEW METHOD}

Figure2 is a schematic diagram summarizing the procedure of constraining the binary stellar and orbital properties, which takes $V_{p}$ and $\left\langle V_{w}\right\rangle$ as two free parameters. Consider an oblate spheroid given by the relation $(x / a)^{2}+(y / a)^{2}+(z / b)^{2}=1$ to describe the overall shape of the circumstellar pattern in a three-dimensional space. The determination of the projection is key in connecting the observed features to the binary properties. The projected shape of the oblate spheroid is obtained by coordinate transformation to the coordinate rotated with the inclination angle $i$. Defining the $x$-axis as the line of nodes (i.e., $x_{\text {proj }}=x$ and $\left.y_{\text {proj }}=y \cos i+z \sin i\right)$ yields the elliptical shape in the projected plane as $\left(x_{\text {proj }} / a_{\text {proj }}\right)^{2}+$ $\left(y_{\text {proj }} / b_{\text {proj }}\right)^{2}=1$ where $a_{\text {proj }}=a$ and $b_{\text {proj }}^{-2}=(\cos i / a)^{2}+(\sin i / b)^{2}$, which simplifies to

$$
\sin ^{2} i=\frac{(a / b)_{p r o j}^{2}-1}{(a / b)^{2}-1} .
$$

Hence, the orbital inclination can be estimated from the observed (projected) axial ratio, given the relation between the aspect ratio of the oblate spheroid and the orbital-to-wind velocity ratio, $a / b=1+2 V_{p} /\left(3<V_{w}>\right)$, as found from our parameter study in KT.

Similarly, simple geometry yields the orbital separation of the binary stars $\left(r_{p}+r_{\text {comp }}\right)$ as a function of the inclination $i$. Figure 3 illustrates the inclined orbit with respect to the plane of the sky, and we obtain the projection effect for the orbital radius of a star to be given by $r_{p, p r o j}=r_{p}\left(1-\sin ^{2} \phi_{p} \sin ^{2} i\right)^{1 / 2}$ and the same for the companion's orbital radius $r_{\text {comp }}$. Therefore, the true orbital separation can be obtained using the observed separation $\left(r_{p}+r_{\text {comp }}\right)_{\text {proj }}$ and position angle PA of the stars from

$$
\begin{array}{r}
r_{p}+r_{\text {comp }}=\frac{\left(r_{p}+r_{\text {comp }}\right)_{p r o j}}{\left(1-\sin ^{2} \phi_{p} \sin ^{2} i\right)^{1 / 2}} \\
\text { where } \tan \phi_{p}=\tan (\mathrm{PA}) / \cos i
\end{array}
$$

and the inclination derived from Equation (11).

On the other hand, the arm spacing $\Delta r_{\text {arm }}$ of the spiral pattern in a binary system is determined by the product of the binary orbital period and the pattern propagation speed in the orbital plane:

$$
\Delta r_{\mathrm{arm}}=\left(<V_{w}>+\frac{2}{3} V_{p}\right) \times \frac{2 \pi r_{p}}{V_{p}} .
$$

This equation shows that the orbital radius $r_{p}$ can be obtained from the pattern spacing $\Delta r_{\text {arm }}$ along the observed longest axis, which refers to a section of the orbital plane, for a given velocity ratio $V_{p} /\left\langle V_{w}\right\rangle$. Using $r_{p}+r_{\text {comp }}$ and $r_{p}$ from Equations (2) and (3), the binary mass ratio $q=M_{p} / M_{\text {comp }}$ corresponding to $r_{\text {comp }} / r_{p}$ is determined. 


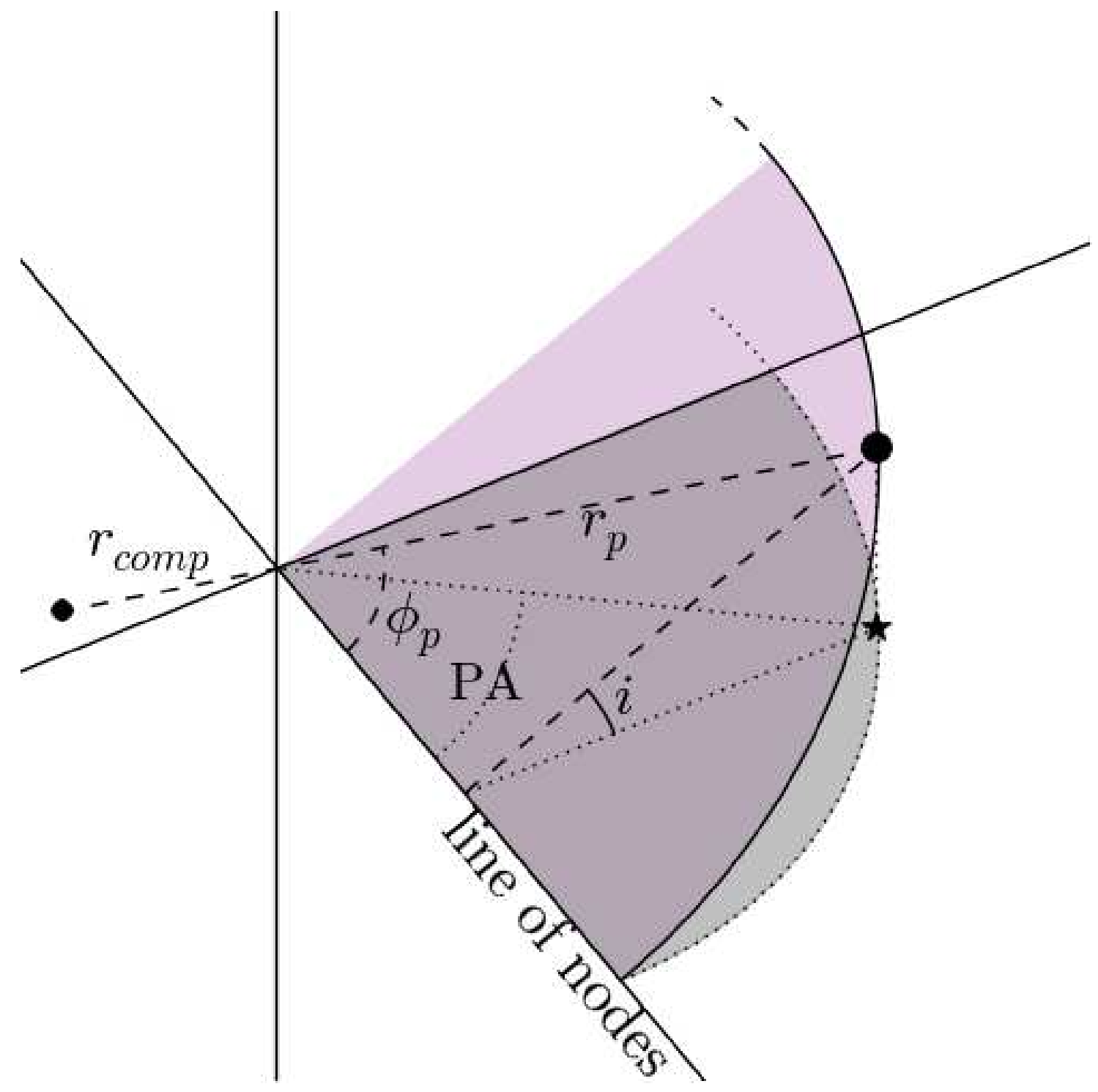

Fig. 3.- Sketch illustrating the binary orbital plane inclined from the plane of the sky with the inclination $i$. The separation of the mass-losing star and companion from the common center of mass are $r_{p}$ and $r_{\text {comp }}$, respectively. The longitudinal angle from the line of nodes is $\phi_{p}$ in the orbital plane, and the projected angle in the plane of the sky is defined by PA. 
Finally, we employ the formula for the force balance in a binary system,

$$
\frac{V_{p}^{2}}{r_{p}}=\frac{G M_{\mathrm{comp}}}{\left(r_{p}+r_{\mathrm{comp}}\right)^{2}}
$$

and replace the quantities related to the companion by the quantities of the mass-losing star using the definition of the $q$ factor, resulting in $M_{p}=q(1+q)^{2} V_{p}^{2} r_{p} / G$. The companion mass is $M_{p} / q$.

\section{CASE STUDY OF AFGL 3068}

\subsection{Observed Properties}

We first find the best fit of an elliptical shape with the dust scattered light pattern of AFGL 3068 (Figure1(b)). The modeled ellipse is characterized by an axial ratio $(a / b)_{p r o j}$ of 1.1 with the major axis in $70^{\circ}$ from the west (i.e., right). Identifying the major axis as the line of nodes of the inclined orbital plane for the reference direction, the position angle (PA) of two point-like sources arranged to the east-west direction is $70^{\circ}$. The observed separation between two sources $\left(r_{p}+r_{\text {comp }}\right)_{\text {proj }}$ is reported (Morris et al. 2006) to be $109 \mathrm{AU}(d / 1 \mathrm{kpc})$, and the interval between ellipses along the major axis, $\Delta r_{\text {arm }}$, is $2350 \mathrm{AU}(d / 1 \mathrm{kpc})$, where $d$ refers to the object distance. The slight deviation from a very good match of the elliptical shape with most parts of the spiral pattern implies that partial circular (or elliptical) rings observed in other AGB CSEs could also contribute to the spiral pattern driven by a binary motion.

\subsection{Parameter Space}

For AFGL 3068, we construct a parameter space, in which $V_{p}$ and $\left\langle V_{w}>\right.$ are spanned, and follow the procedure summarized in Figure 2 to calculate the corresponding orbital properties of the binary system. Figure 4 shows the result, restricting the parameter space that satisfies the conditions for AFGL 3068 as a carbon-rich AGB star in a binary system. First, the gas velocity cannot exceed $\left\langle V_{w}\right\rangle+V_{p}$ in any direction (KT), which yields $<V_{w}>+V_{p} \gtrsim 14 \mathrm{~km} \mathrm{~s}^{-1}$ with the line-of-sight velocity of $14 \mathrm{~km} \mathrm{~s}^{-1}$ defined from the half width of spectral lines (Loup et al. 1993). Second, the mass of a carbon star such as AFGL 3068 is theoretically constrained to be less than $4 M_{\odot}$ since more massive stars ( 4 to $8 M_{\odot}$ ) are sufficiently hot at the base of their convective envelopes to activate the so-called "hot bottom burning" process, which significantly burns the envelope carbon (Boothroyd et al. 


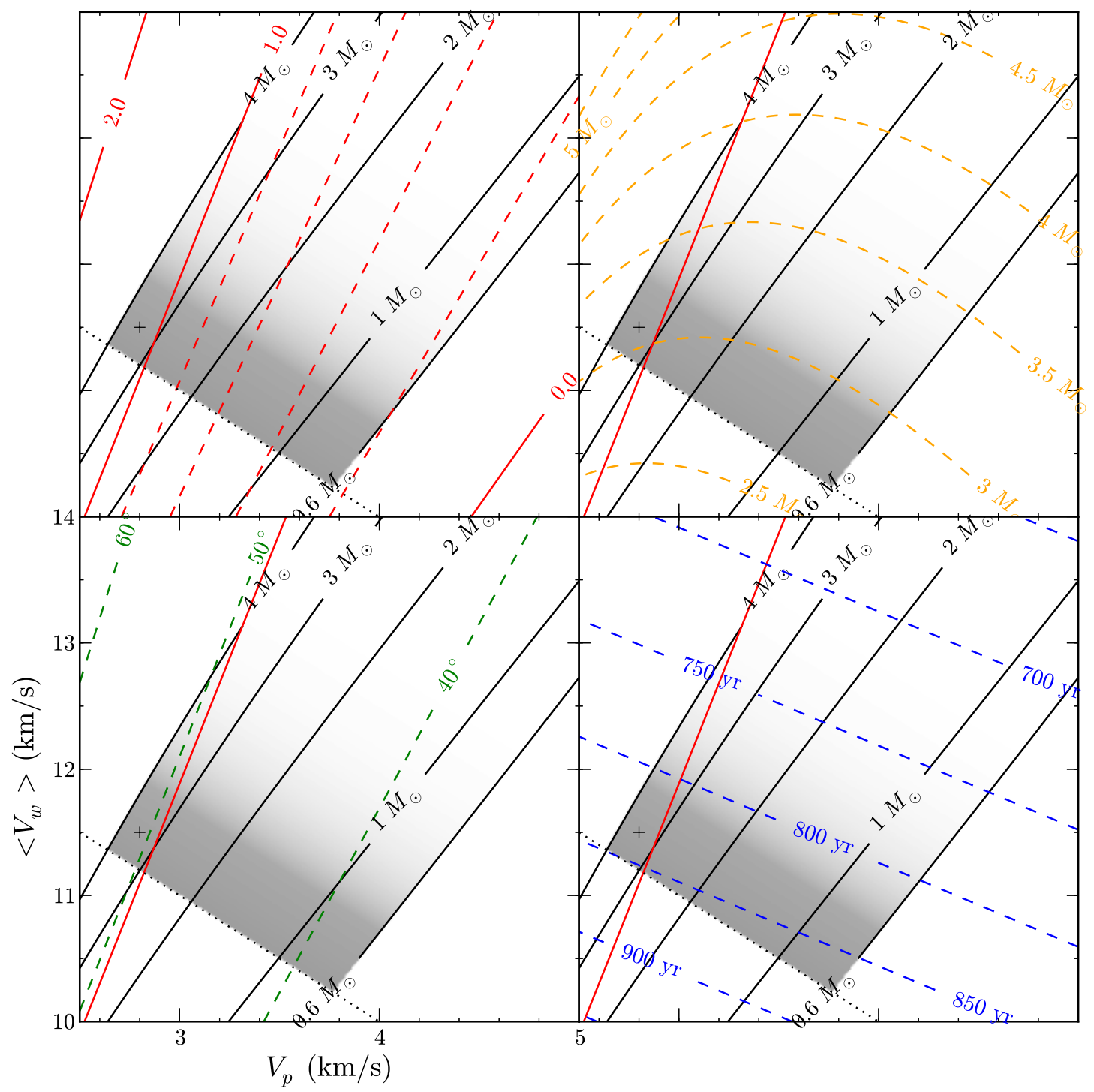

Fig. 4.- Model parameter space for the speeds of orbital motion $V_{p}$ and outflowing wind $<$ $V_{w}>$ of the mass-losing star in AFGL 3068. An expansion velocity obtained from molecular line emissions (Loup et al. 1993) provides a constraint, $\left\langle V_{w}\right\rangle+V_{p} \geq 14 \mathrm{~km} \mathrm{~s}^{-1}$ (dotted line), and the mass of the AGB carbon star (Boothroyd et al. 1993, 1995) can be 0.6-4M. (black solid line), confining the parameters for AFGL 3068 into the gray-colored region. Considering the carbon star to be more massive than its companion, the parameter space for AFGL 3068 is further restricted to above the red line for the mass ratio of 1 . The + sign corresponds to the parameters for which a hydrodynamic simulation was carried out for comparison to observations (Figure1). Top left. The mass ratio between the carbon star and companion of 0,1 , and 2 (red solid lines) and 0.2, 0.4, 0.6, and 0.8 from bottom (red dashed lines). Top right. The mass of the companion with an $0.5 M_{\odot}$-interval (yellow dashed lines). Bottom left. The inclination angle of the orbital plane with respect to the plane of the sky with a ten-degree interval (green dashed lines). Bottom right. The orbital period of binary stars with a 50-year interval (blue dashed lines). 
1993, 1995). Third, we take the lower limit of the carbon star as $0.6 M_{\odot}$, which is the mean mass of white dwarfs (Madej et al. 2004).

These three constraints place the AFGL 3068 system within the gray-colored area in Figure4, As a result, the mass of the companion is constrained to be greater than $2.6 M_{\odot}$ with the binary mass ratio $q=M_{p} / M_{\text {comp }}$ greater than 0.2 . In addition, the inclination angle of the system is constrained to lie within approximately $40^{\circ}-50^{\circ}$. Finally, the orbital period is found to be less than 870 years, and the orbital speed of the carbon star in the range of $2.5-4 \mathrm{~km} \mathrm{~s}^{-1}$. Assuming an accuracy of the distance measurement to be $20 \%$, the companion mass is estimated to be greater than 2.1 to $3.2 M_{\odot}$. If the line-of-sight velocity of the wind is $16 \mathrm{~km} \mathrm{~s}^{-1}$ (De Beck et al. 2010), the estimated companion mass is larger than 2.8 or $4.2 M_{\odot}$ at distance of 0.8 or $1.2 \mathrm{kpc}$. The combination of a high wind velocity $\left(16 \mathrm{~km} \mathrm{~s}^{-1}\right)$ and large distance $(1.2 \mathrm{kpc})$ results in a large companion mass, leading to an implausible mass $>4 M_{\odot}$, for which the companion should have evolved more rapidly than the carbon star.

As more massive stars evolve more rapidly, the carbon star is likely to be more massive than its companion $(q>1)$, although the mass ratio $q$ could be less than unity because the carbon star is in the phase of losing mass at a high rate $\left(>10^{-5} M_{\odot} \mathrm{yr}^{-1}\right)$ (Jones et al. 1978; Volk et al. 1992; Woods et al. 2003; Loup et al. 1993; Ramstedt et al. 2008; Ladjal et al. 2010). In the case that the total mass lost from the carbon star is not too extensive, the parameters can be further constrained into a small parameter space, indicating individual stellar masses of $3-4 M_{\odot}$, speed of $2.5-3.5 \mathrm{~km} \mathrm{~s}^{-1}$ with the orbital period of $700-850$ years in the plane inclined by approximately $50^{\circ}$.

\subsection{Hydrodynamic Model}

Within the limited area in the parameter space where AFGL 3068 can lie, we select a point (plus sign in Figure4) to compare the corresponding hydrodynamic model with the observed pattern in the CSE of AFGL 3068. Figure1 indeed shows that the model shows good agreement with the scattered light image of AFGL 3068. In particular, the Archimedes spiral characterized by having a constant spacing (Mauron \& Huggins 2006) passes inside the intensity peak positions in the northerly direction, while the spiral of our model better traces the positions of the pattern. The blurred patterns seen in the northwest and south closely resemble the knotty structures appearing along the line of nodes, which form from the overlap of the two structures generated by the orbital motions of individual stars (KT).

The modeled binary system is characterized by a mass-losing star of mass $M_{p}=3.5 M_{\odot}$, velocity $V_{p}=2.8 \mathrm{~km} \mathrm{~s}^{-1}$, and orbital radius $r_{p}=78 \mathrm{AU}$, and a companion of mass $M_{\text {comp }}=$ 
$3.1 M_{\odot}$, velocity $V_{\text {comp }}=3.2 \mathrm{~km} \mathrm{~s}^{-1}$, and orbital radius $r_{\text {comp }}=88 \mathrm{AU}$. The wind velocity $V_{w}$ was set to be $10.9 \mathrm{~km} \mathrm{~s}^{-1}$ at $30 \mathrm{AU}$ distance from the mass-losing star. The gas flow evolves to a quasi-steady state fluctuating about $11.5 \mathrm{~km} \mathrm{~s}^{-1}$ with a standard deviation of $0.67 \mathrm{~km} \mathrm{~s}^{-1}$. To achieve a temperature profile of $r^{-0.83}$ as determined from an analysis of the CO molecule excitation (Woods et al. 2003) for a density profile of $r^{-2}$, the adiabatic index $\gamma$ of the wind material is set to be 1.4 with the initial temperature of $1540 \mathrm{~K}(\mu / 2)$ at $30 \mathrm{AU}$ distance from the mass-losing star, where $\mu$ indicates the molecular weight. Figure5 shows that the resulting temperature profile indeed follows the expected power law with the index of -0.83 and has an average value of approximately $60 \mathrm{~K}(\mu / 2)$. Additional heating in the post-shock region and cooling via molecular line transitions are not considered as the timescales in the CSEs of AGB stars are comparable (Edgar et al. 2008). The density power law index is -2.2 in the resulting configuration. The volume and column density contrasts correspond to a factor of $10-30$ and 2-5, respectively. We note that the density contrast in the model with $\gamma=1.4$ is approximately constant, while the contrast in an isothermal model decreases rapidly with distance from the system center $(\mathrm{KT})$. The dependencies of the overall shape and morphology of the spiral pattern on the orbital-to-wind velocity ratio are insensitive to the value of $\gamma$. The mass loss rate taken as $4 \times 10^{-5} M_{\odot} \mathrm{yr}^{-1}$ (Olivier et al. 2001) provides the scale for the magnitude of the density, but not the density contrast. The resulting column density toward the star is of order of $10^{23} \mathrm{~cm}^{-2}$, which varies by a factor of a few with the inclination angle of the system.

The observed pattern thickness of AFGL 3068 is thinner than the arm thickness of the modeled column density distribution, and is rather comparable to the density distribution in the mid-plane of the sky. Because of the curved geometry of the spiral-shell pattern, the shocked edges of the pattern at different layers are projected into different positions in the plane of the sky, resulting in a broader arm for the column density. It may imply that the observed dust scattered light is optically thick so that the observed image refers to only part of the pattern in the line of sight. This is consistent with the high optical depth derived from the spectral energy distribution fitting (Ladjal et al. 2010). The observed pattern thickness may also depend on the observed waveband. In particular, the size distribution of the dust grains may be affected, resulting from coagulation or shattering via grain-grain collisions immediately after encountering the shock with a velocity dispersion of a few $\mathrm{km} \mathrm{s}^{-1}$ (Jones et al. 1996). As a consequence, the variation of the size distribution may affect the intensity across the shock pattern at the observed wavelength. Small grains with a size less than $0.045 \mu \mathrm{m}$ tend to strongly couple with the gas, while larger grains are characterized by a different distribution as they are more resistant to the drag force (van Marle et al. 2011). The probable large population of small grains near the shock increases the scattering cross section with the large surface-to-volume ratio of the small grains, resulting in an increase of 


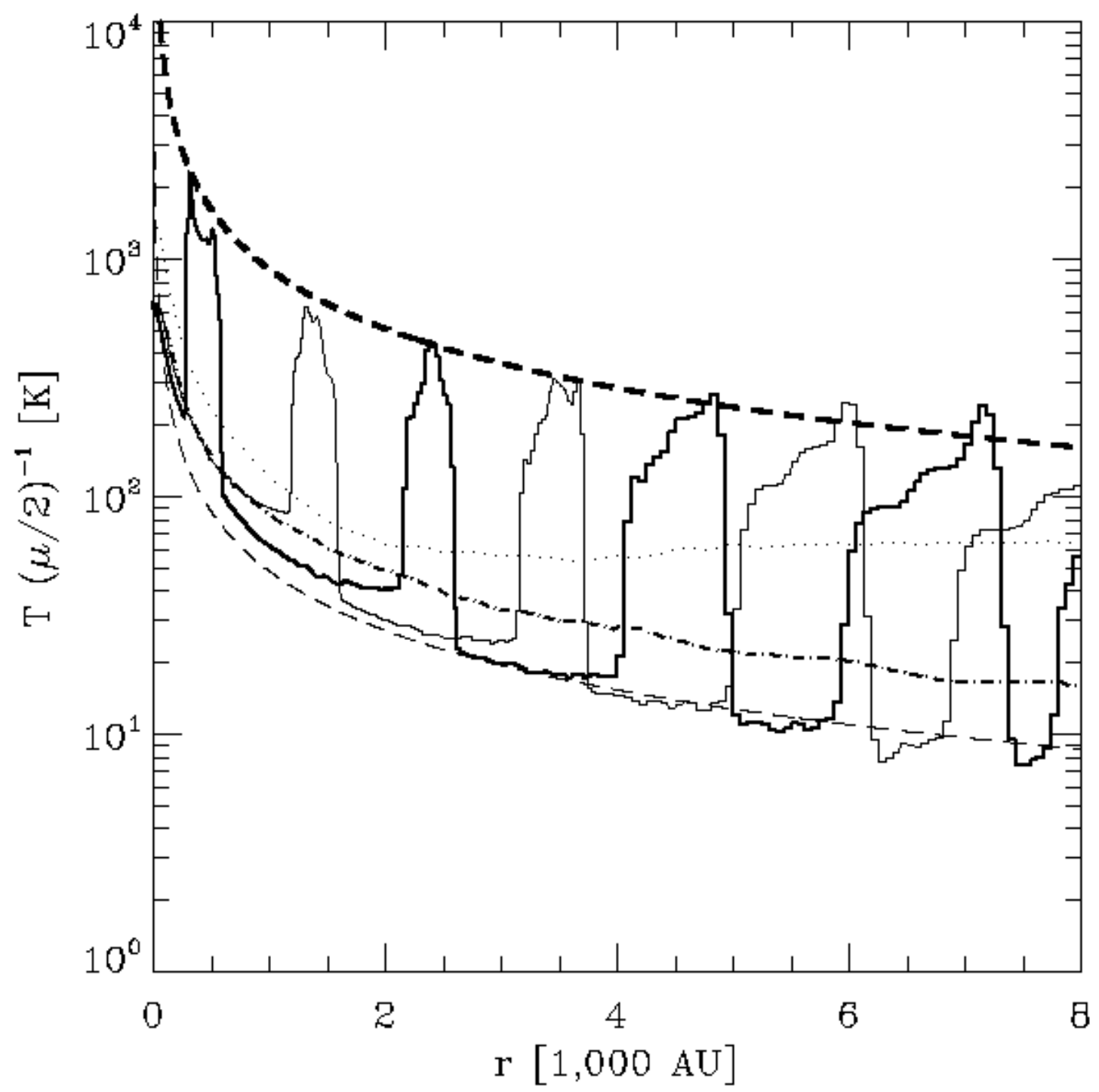

Fig. 5.- Temperature profile in the outflowing CSE surrounding the binary system along two arbitrary-chosen directions in the orbital plane (thick and thin solid lines) in the hydrodynamical model. The peak temperature within the spiral arm has the value $2.8 \times 10^{5} \mathrm{~K}(\mu / 2)(r / \mathrm{AU})^{-0.83}$ (thick dashed line), while the inter-arm temperature approximates to $1.5 \times 10^{4} \mathrm{~K}(\mu / 2)(r / \mathrm{AU})^{-0.83}$ (thin dashed line). The temperature along the orbital axis (dot-dashed line) and the value averaged over three dimensional space (dotted line) are consistent with the result of a radiative transfer modeling for the observed CO line emission (Woods et al. 2003). 
the scattered light intensity at the corresponding wavelengths.

Our model assumes circular motion of the binary stars. However, previous calculations for eccentric orbits do not show a noticeable change in the overall shape outside the second

turn of the spiral pattern (He 2007; Raga et al. 2011), suggesting that our model for the large scale shape of the pattern driven by circular orbits is a good approximation even for the case of non-circular orbits. Although a highly eccentric orbit $(e \gtrsim 0.8)$ causing substructure in the inter-arm region (prominent between the innermost turns) may be responsible for the extra structure found in the observed image between first and second turns in the northwest and southeast, a circular orbit model also exhibits a weak density enhancement close to the observed substructure. This substructure can also possibly be affected by local phenomena around the companion (e.g., the formation of an accretion disk and radiation pressure effects).

\section{Conclusion}

We have developed a new method to estimate the stellar and orbital characteristics of binary systems with an AGB component using information gleaned from the properties of a spiral pattern in the CSE. As a first application, we have provided constraints on the inclination angle, orbital period, and companion mass in the binary system AFGL 3068 based on the pattern shape of AFGL 3068 seen in dust scattered light. With molecular line observations, additional kinematic information on the velocity structure of the pattern will provide further constraints on the system properties.

The potential for estimating system parameters with the method proposed in this paper is promising and can be useful in providing fundamental input about systems where asymmetries in CSEs are observed. As such one may be able to use these observational data to place constraints on the input parameters and prescriptions used for the evolution of systems in population synthesis studies of detached binary systems (see Politano \& Taam 2011). It is likely that the application of this method to other systems, which we can anticipate will be found with very sensitive and high resolution observations using e.g., the Atacama Large Millimeter/submillimeter Array, will provide a greater statistical basis for determining the incidence of such evolved binary systems in the total stellar binary population.

This research is supported by the TIARA in the ASIAA. We are grateful to A\&A, N. Mauron and P. J. Huggins for their agreement to allow us to reprint their published HST image. H.K. thanks H. Hirashita, P. Koch, I-T. Hsieh, members of ICSM and CompAS groups in ASIAA for discussions and comments. 


\section{REFERENCES}

Boothroyd, A. I., Sackmann, I.-J., \& Ahern, S. C. 1993, ApJ, 416, 762

Boothroyd, A. I., Sackmann, I.-J., \& Wasserburg, G. J. 1995, ApJ, 442, L21

De Beck, E., Decin, L., de Koter, A., et al. 2010, A\&A, 523, A18

Edgar, R. G., Nordhaus, J., Blackman, E. G., \& Frank, A. 2008, ApJ, 675, L101

Habing, H. J. 1996, A\&A Rev., 7, 97

He, J. H. 2007, A\&A, 467, 1081

Huggins, P. J. 2007, ApJ, 663, 342

Jewell, P. R., \& Snyder, L. E. 1982, ApJ, 255, L69

Jones, A. P., Tielens, A. G. G. M., \& Hollenbach, D. J. 1996, ApJ, 469, 740

Jones, B., Merrill, K. M., Puetter, R. C., \& Willner, S. P. 1978, AJ, 83, 1437

Kim, H. 2011, ApJ, 739, 102

Kim, H., \& Taam, R. E. 2012a, ApJ, 744, 136

Kim, H., \& Taam, R. E. 2012b, ApJ, accepted (KT)

Ladjal, D., et al. 2010, A\&A, 513, 53

Lebofsky, M. J., \& Rieke, G. H. 1977, AJ, 82, 646

Loup, C., Forveille, T., Omont, A., \& Paul, J. F. 1993, A\&AS, 99, 291

Madej, J., Należyty, M., \& Althaus, L. G. 2004, A\&A, 419, L5

Mastrodemos, N., \& Morris, M. 1999, ApJ, 523, 357

Mauron, N., \& Huggins, P. J. 2006, A\&A, 452, 257

Morris, M., Sahai, R., Matthews, K., et al. 2006, Planetary Nebulae in our Galaxy and Beyond, 234, 469

Olivier, E. A., Whitelock, P., \& Marang, F. 2001, MNRAS, 326, 490

Politano, M., \& Taam, R. E. 2011, ApJ, 741, 5 
Raga, A. C., Cantó, J., Esquivel, A., Huggins, P. J., \& Mauron, N. 2011, in Asymmetric Planetary Nebulae 5 Conference, ed. A. A. Zijlstra, F. Lykou, I. McDonald, and E. Lagadec (Jodrell Bank Centre for Astrophysics, Manchester), 185

Ramstedt, S., Schöier, F. L., Olofsson, H., \& Lundgren, A. A. 2008, A\&A, 487, 645

Silverglate, P., Terzian, Y., Zuckerman, B., \& Wolff, M. 1979, AJ, 84, 345

Soker, N. 1994, MNRAS, 270, 774

van Marle, A. J., Meliani, Z., Keppens, R., \& Decin, L. 2011, ApJ, 734, L26

Volk, K., Kwok, S., \& Langill, P. P. 1992, ApJ, 391, 285

Woods, P. M., Schöier, F. L., Nyman, L.-Å., \& Olofsson, H. 2003, A\&A, 402, 617

Zhang, Y., Kwok, S., \& Nakashima, J.-i. 2009, ApJ, 700, 1262 